\title{
Design and Implementation of Measurement System of Mechanical Parts with Multihole Based on Machine Vision
}

\author{
Chen Qiyu, Wang Yu, Wu Zhiheng, Tong Jigang, Mo Juexian
}

Guangdong Institute of Intelligent Manufacturing, Guangzhou, China

\section{Email address:}

Walker2000@126.com (Chen Qiyu),397007595@qq.com (Wang Yu),wuzhiheng23@163.com (Wu Zhiheng),

tongjigang@163.com (Tong Jigang),mojuexian520@163.com (Mo Juexian)

\section{To cite this article:}

Chen Qiyu, Wang Yu, Wu Zhiheng, Tong Jigang, Mo Juexian. Design and Implementation of Measurement System of Mechanical Parts with Multihole Based on Machine Vision. Journal of Electrical and Electronic Engineering. Vol. 6, No. 2, 2018, pp. 65-70.

doi: $10.11648 /$ j.jeee.20180602.15

Received: June 22, 2018; Accepted: August 4, 2018; Published: August 13, 2018

\begin{abstract}
The efficiency of the measurement of mechanical parts with multihole is needed to be improved in the information era, while the size measurement of mechanical parts relying mainly on manual detection currently with very low efficiency. To meet the repuiremant of equipment manufacturing automation, the efficiency of the measurement of mechanical parts should match the speed of the line. As a new technology to solve the measurement problem of the objects with irregular shape (such as multihole), machine vision provide a more effective way. To build a measurement system of mechanical parts with multihole, there are many relevant aspects to be considered, such as the choices of hardware, software development, algorithm design, ect. Image processing is one of the most important steps to build a successful visual system, which usually consists of image preprocessing, image segmentation, feature extraction and defect classification. The design and implementation of measurement system of mechanical parts with multihole based on machine vision will be discussed in this paper. The experiment results show that the improved algorithm can effectively filter the noise of surface images, which make the outline of the hole can be tested out easier. The system in this paper not only ensures the measurement precision of the pore diameter of the workpiece, but also realizes the measurement of the pore diameter of the workpiece at the same time.
\end{abstract}

Keywords: Machine Vision, Mechanical Parts, Size Measurement

\section{Introduction}

With the rapid development of the equipment manufacturing industries, such as aviation industry, automobile industry, etc., the demand for relevant mechanical parts of the equipments with complex structure is very great. As a result, more and more mechanical parts with irregular shape are manufactured, such as the mechanical parts with multihole. Before assembling mechanical parts with holes, the holes must be detected to ensure that the holes meet the technical requirements of assembly. It is to say, the size measurement of them have to be done first. At the same time, the efficiency of the measurement of mechanical parts should match the speed of the equipment manufacturing. For example, stamping parts size measurement is the key link in production. The traditional measuring means (such as calipers, gauge, screw micrometer, etc.) is easy to operate, but with big workload, low measuring efficiency and being easily affected by man-made factors. It cannot be used for online measurement, can not meet the requirement of the modern mass, automated measurement [1].

China is a large manufacturing country, which produces a large number of industrial products every day. Users and manufacturers are increasingly demanding the quality of products [2]. In recent years, in order to improve the efficiency of the size measurement of mechanical parts, lots of measurement technology and systems based on machine vision technology are developed. Machine vision is the technology and methods used to provide imaging-based automatic inspection and analysis for applications such as automatic inspection, process control, and robot guidance, usually in industry. There are many advantages when doing the size measurement base on machine vision, such as non-contact, fast and efficient. The demand of intelligent visual inspection systems aimed at ensuring the high quality in production lines is increasing. Different measurement 
technology and systems based on machine vision technology have been development around the world. Zhang Tian et al. applying machine vision for bend space parameter measurement, under the condition of specific measurements to solve the large size complex elbow space parameter measurement [3]. Wang Zhongfei et al. applying machine vision to measure the thickness of microchip, reduced the measurement system cost, and improved the measurement speed [4]. Marco San Biagio et al. present a computer vision system that, using only images, is able to address two main problems: (i) model checking: automatically check whether a component meets given specifications or rules, (ii) visual inspection: defect inspection on irregular surfaces, in particular, decolourization and scratches detection. [5]

But as to the size measurement of mechanical parts with multihole, there are little literature review in this field. To improve both the efficiency and accuracy of the measurement of mechanical parts with multihole, this paper introduces a measurement system based on machine vision. In this paper, the overall framework of the system is proposed first, and then the hardware selection is discussed. Image processing is the most important part of the system development. Because multihole is the key feature of the workpiece, so it is more difficult for the measure system to complete the measure task. Sometimes there are more than 3 holes within the workpiece, even up to 10 holes with different size. Each hole has its own characteristics. The holes must be identified simultaneously, so the measuring system must accurately identify each hole separately. In order to get accurate information, image filtering, image segmentation, feature extraction should be included. In order to solve the problem of image processing, a special algorithm is designed. Finally, an experimental summary is given.

\section{Overall Framework of the System}

The measurement system consists of two parts: the hardware and the software, shown in figure 1.

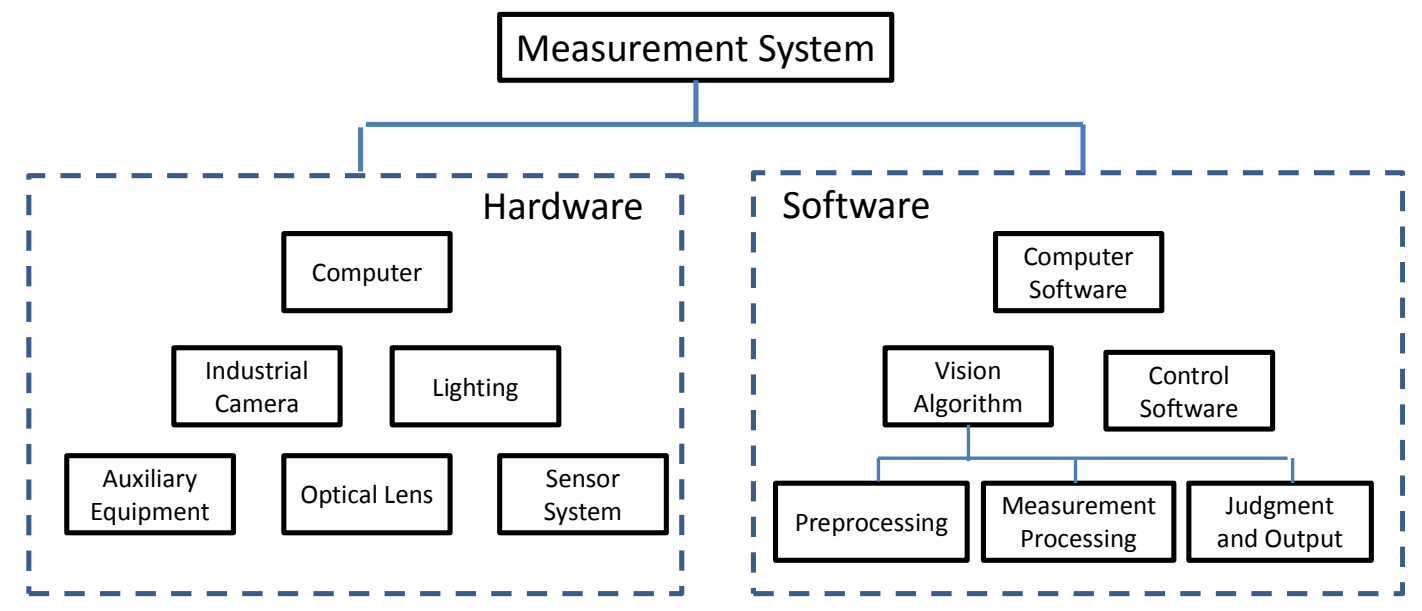

Figure 1. Overall framework of the system.

The hardware of the measurement system mainly includes the sensor system, optical lens, lighting, industrial camera, computer and the auxiliary equipment. The software of the system mainly includes computer software, control software, and vision algorithm. The vision algorithm is the special software that deal with the image of the mechanical parts, usually including the preprocessing, measurement processing, judgment and output module.

In fact, the overall framework of the measure system is a normal framework. It is very similar to other machine vision application systems. The suitable hardware selection is the basic job, because the researchers have to build a real testing environment. In fact, the biggest challenge is developing the software.

When the workpiece triggers the image acquisition sensors, the industrial camera will capture the surface images of workpiece. The lighting helps the industrial camera to get higher quality image. The image processing module doing the edge contour feature extraction, identification and measurement through a series of mathematical morphology processing.

\section{Discussion of the Choices of Hardware}

How hardware is designed depends on the given specification of the measurement. The actual vision system usually selects different industrial cameras to collect images according to color requirements and resolution. In this paper, the mechanical part used for testing is made of steel. Industrial camera requires high image quality, high transmission rate and standard interface. In order to meet the test requirements, the industrial camera with gmt200-h high frame rate of 2 million pixels is used.

Different light sources have their distinguishable effects on the quality of workpiece images acquisition. This system use the LED (light emitting diode) backlight source while LED has many excellent properties, such as long life, energy-saving, better stability. By adjusting the position of the light source and reducing the reflected light of the workpiece, a good image acquisition quality can be obtained. Industrial camera and LED backlight source are shown in figure 2 . 

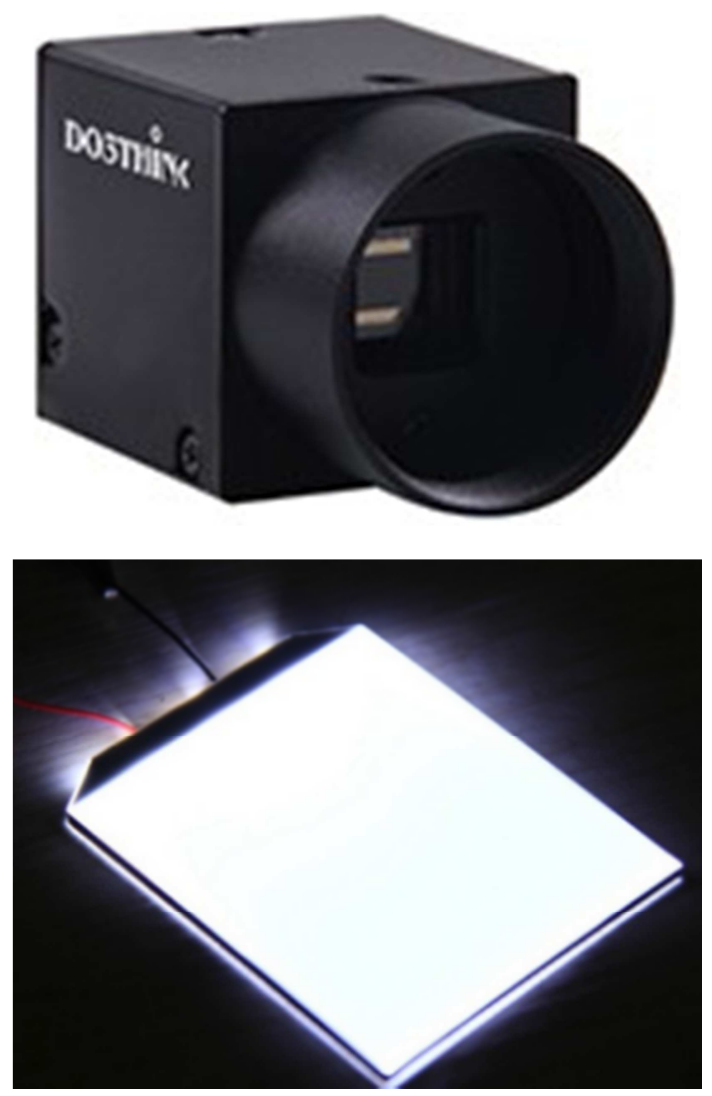

Figure 2. Industrial camera and LED backlight source.

The stability and reliability of the measurement system is the premise of automation and intellectualization of the assembly line of mechanical parts. This system uses different sensors in this measurement system. The sensor can trigger the image acquisition part at the beginning. Multi-sensor fusion not only guarantees the accuracy of system detection, but also makes the detection more intelligent. The parameters of each sensor are listed below, shown in table 1 .

Table 1. Sensors and their parameters.

\begin{tabular}{lll}
\hline Name of Sensor & Model & Main parameters \\
\hline Photoelectric sensor & GTD-2 & Distance $30-150 \mathrm{~mm}$ \\
Displacement transducer & KTC-1 & $\pm 0.01 \mathrm{~mm} ; 50 \sim 110 \mathrm{~mm}$ \\
Wireless sensor & JZH-0 & $25 \mathrm{~mA}, 2000 \mathrm{~m}$ \\
\hline
\end{tabular}

\section{Image Processing}

\subsection{Image Acquisition}

Image processing usually consists of image preprocessing, image segmentation, feature extraction and defect classification [6]. Image acquisition is the first step for the vision system to obtain visual information.

The acquisition of high quality images can make the images easy to process, and make the recognition and positioning results more accurate as well. Usually both the working environment and the equipment themselves affect the quality of image acquisition. The image contains various noises and uneven brightness [7]. In order to obtain higher quality image, This system use industrial camera and LED backlight source.

\subsection{Image Feature Enhancement}

Image enhancement can be used to make previously unclear images clear, or to blur previously clear images to meet the needs of some special analysis [8]. For the colour image contains a large amount of information, using colour image is more ideal to some extend. But at the same time it raises higher request on the processing speed of image for the processing system, because colour image texture feature is more complex. The image texture of the workpicec in this paper is relatively simple. Using the image gray level information can not only complete the recognition of image features, but also improve the speed of image processing.

Feature enhancement can improve the intensity of feature signal in multihole feature images. The method of image enhancement is to add some information or transform data to the original image by means of certain means. It can selectively highlight the features of interest in the image or suppress some unnecessary features in the image, so that the image and the visual response characteristics are matched. The gray image feature enhancement is carried out in this paper. The grayscale adjustment makes the feature information of multihole feature images more obvious.

\subsection{Image Filtering}

Due to the digital image acquisition is much different from that of the traditional picture, it is easy to be disturbed in image acquisition and transmission links. As a result, the digital image obtains irrelevant information while would affect the image recognition. In order to improve the image quality, the corresponding image preprocessing methods are used to eliminate the noise interference. Image filtering is an effective method to eliminate noise. Its theoretical basis is image convolution. For example, the low pass filtering can remove noise, while the high pass filtering can enhance high frequency signals such as edge, so as to make the fuzzy image more clear. The corresponding processing time of each filtering method is different, and the processing effect is different too. Each filter has its own advantages and disadvantages, so it is necessary to select an appropriate filter in the actual filtering process. In order to obtain the edge information of workpiece, the edge information of workpiece image needs to be saved while filtering.

High quality image is very important for the system to analyse the characteristics of the workpiece in the actual working condition. Usually the quality of original image is not high enough to be processed directly. Image filtering is a effective way to improve the quality of the image. Selecting an appropriate filtering can not only eliminate the noise in the image, but also avoid blurring the edge contour and line of the image.

\subsection{Image Threshold Segmentation}

Common shape extraction techniques include threshold segmentation and background subtraction. The background 
subtraction method assumes that the background is accurately known. The foreground image can be obtained by subtracting the current image from the background image. When the camera collects images, it may be affected by lighting, which will cause the background in the current image to be significantly different from the previous one. The threshold segmentation is applied to extract useful shape information. Threshold segmentation is also called binarization. Set a grayscale threshold for pixels in a grayscale image to extract useful grayscale information. The value of global threshold segmentation needs manual setting and poor adaptability. Methods with adaptive threshold include Otsu's method and adaptive global threshold method. The basic idea of Otsu's method is to divide the image gray histogram into two groups at a certain threshold value. When the variance between the two groups is maximum, the corresponding threshold is determined to be the selected threshold value. In this section, image segmentation is performed by setting threshold manually. The resulting threshold segmentation image is shown in figure 3 .

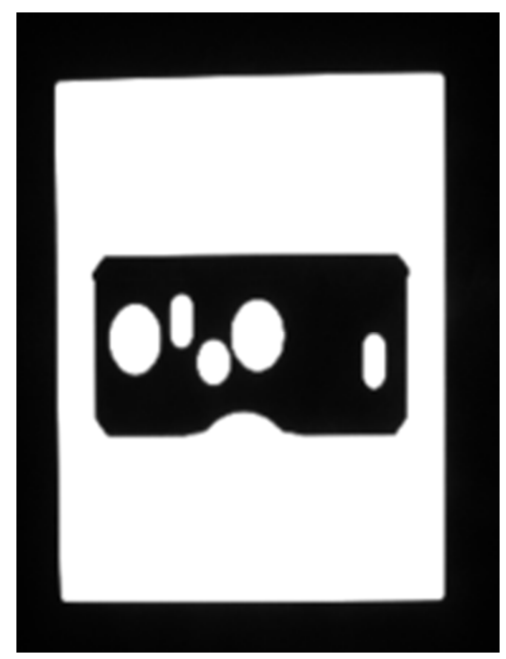

Figure 3. Image threshold segmentation.

\subsection{Image Edge Extraction}

Edge is an important feature of object recognition, with certain details of image. At the same time, the image contour can be recognized by extracting the edge of the image. The edge of the image corresponds to the most prominent change in the local pixel value of the image. The image can be regarded as the sampling point array of image intensity continuous function. The gradient is the measure of function change, so the significant change of image pixel value can be detected by the discrete approximation function of function gradient. More commonly used edge detection methods include Canny operator, Prewitt operator, Sobel operator, etc. Considering the characteristics of multihole workpiece, Canny operator is adopted in this paper.

Edge contains complex information of the target object, which can extract some important features of the image in image segmentation and analysis. Machine vision has the characteristics of high measurement precision, fast response speed and full-court testing [9]. The edge is the set of points where the grayscale value changes suddenly in the image. The purpose of edge detection is to obtain the contour lines and feature points of the target in the image. With the rapid development of machine vision and digital image processing, edge detection algorithms are widely used. Especially in workpiece size detection, edge detection algorithm is an essential link. Therefore, the detailed study of edge detection technology has important theoretical and practical significance. In the workpiece size measure system, if the accurate edge information can be obtained, the pixel size and actual size of the workpiece can be calculated by using the distance between the feature points of the edges. Image contour edge shown in figure 4.

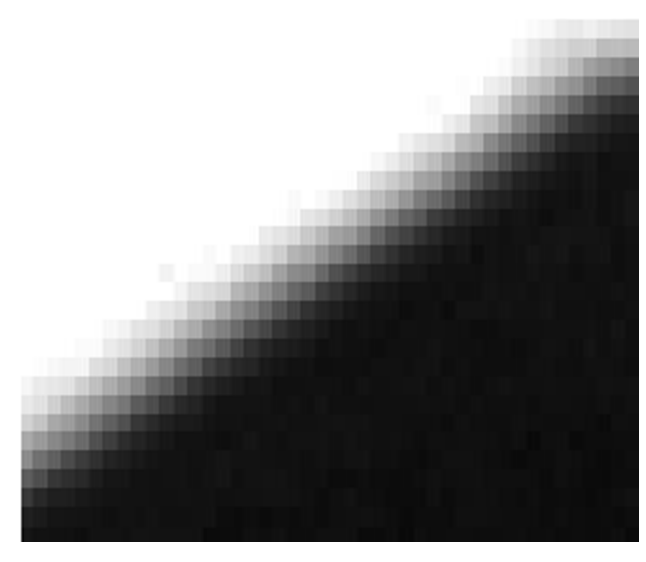

Figure 4. Image contour edge.

Edge feature is refers to the edge of the image within a certain neighborhood grayscale value where the total number of pixels mutation, is the most important place for image recognition, pattern recognition, edge detection, parameter calculation of image processing as well. Only the edge points are detected first, the system can make the follow-up work going on. At the same time, the location precision of edge points directly affect the final image processing result. Obviously, the image edge extraction is the key step in the size measurement of the workpicec. The image unprocessed edge profile is shown in figure 5 .

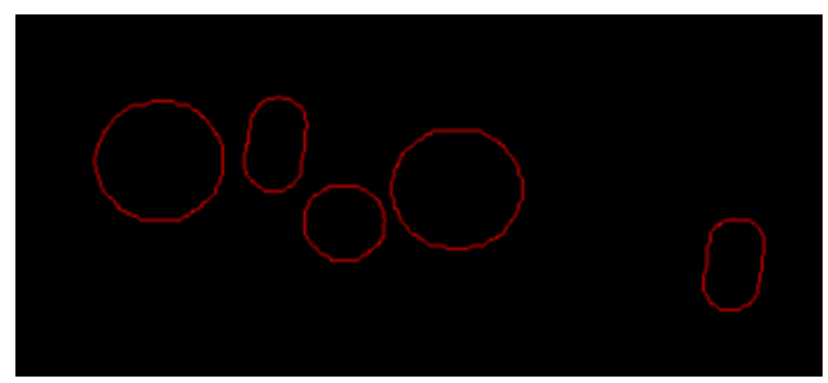

Figure 5. Image unprocessed edge profile.

With such significance in machine vision, image edge has been the focus by many scholars for a long time. The edge of an image can be described with direction and min value. The 
complex structure of workpiece and the complicated measurement process make it difficult to improve the efficiency [10]. Generally speaking, along the direction of the edge, the gray value changes little, while in the vertical direction, the gray value changes greatly. It is very difficult to describe the edge by pure mathematics. In order to express the edge of the image more clearly, the edge of the image can be described from the following four aspects:

(1) position of the edge: that is, the coordinate position of the edge point, refers to the pixel coordinates of the point in the image where the gray value changes;

(2) edge direction: the edge direction of a certain point in the image refers to the direction that is tangent to the target edge at that point;

(3) normal direction of the edge: perpendicular to the edge direction;

(4) edge intensity change: refers to the local image intensity change in the normal direction of the edge in a neighborhood of the target edge point.

The image fitted measuring profile is shown in figure 6 .

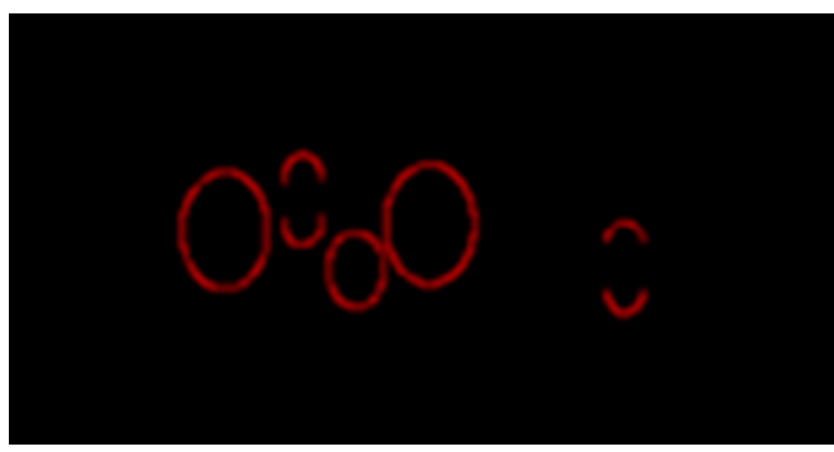

Figure 6. Image fitted measuring profile.

\section{Experiment and Analysis}

In this paper, the workpiece used for testing is one of mechanical parts of automobile. Both the aperture and the coordinate of the center of a circular hole of the workpiece are needed to be measured.

Different parts of the workpiece have their own features. In order to process images, it must understand regional features, grayscale values, and contour features, etc.. As to the measurement of mechanical part with multihole, both the characteristics of the main edge contour features of holes, and the variation of the edge contour have to be judged by the direction of the gradient. At the same time, the direction and boundary of the gradient shows different characteristics, so the strength of the edge contour can be determined by gradient mode change information.

Because the workpiece is manufactured by machines, its surface is not so smooth. When the industrial camera captures the surface image, the noise are also be gathered at the same time. The noise not only influences the detection of the edge contour, but also affects the measurement and detection of the hole aperture in the later stage. To get the higher quality image of the workpiece, developing the new algorithm, instead of the general methods, is needed. The images below show the difference. The middle image is the the result using the general edge contour detection method, while the right image is the other result using the improved algorithm.
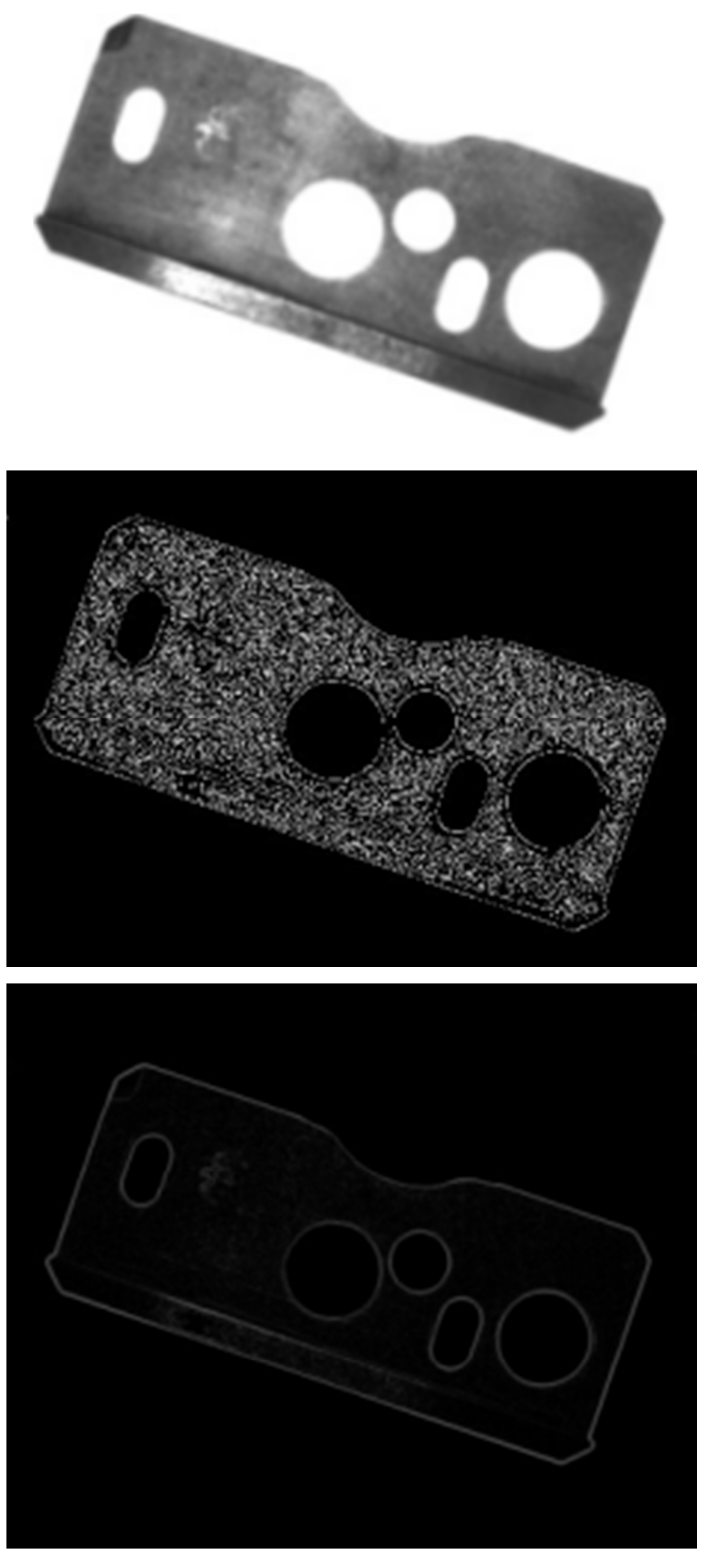

Figure 7. Experimental results of edge contour detection operator.

From the above experimental results, it can be found that the improved algorithm can effectively filter the noise of surface images. With the higher quality image, the outline of the hole can be tested out easier. The interfaces of the measurement system are shown in figure 8 . 


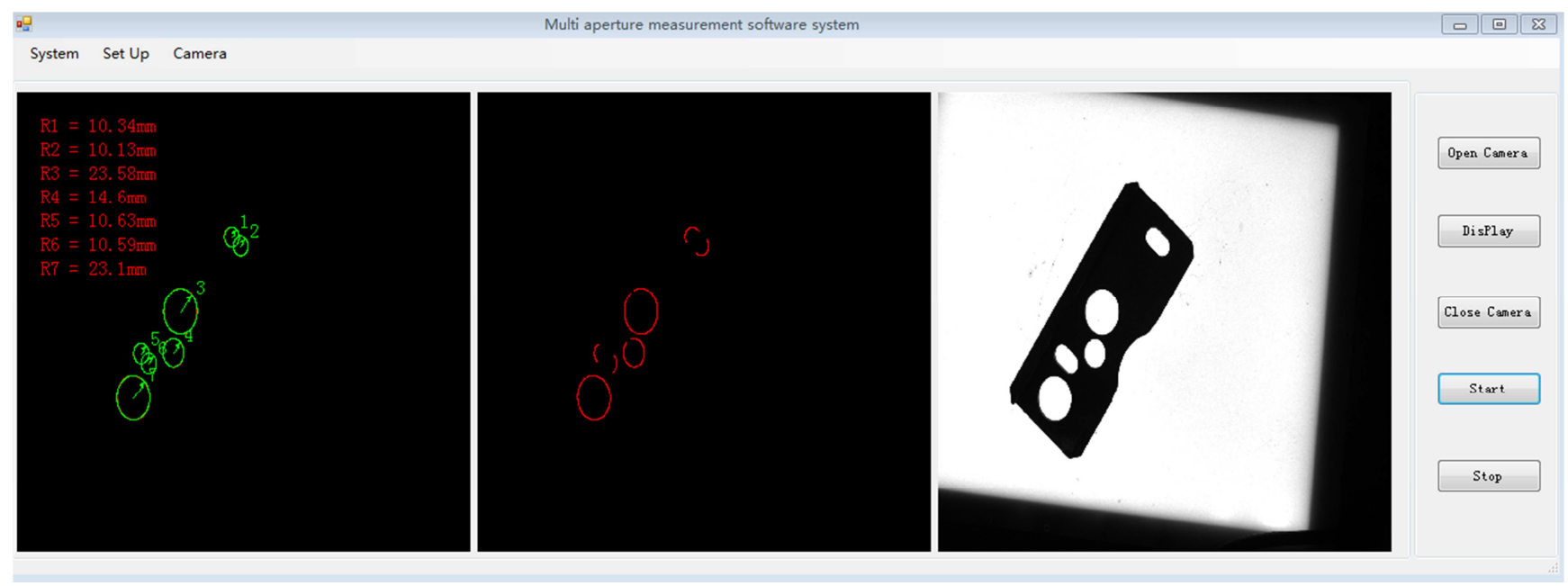

Figure 8. The interfaces of the measurement system.

\section{Conclusion}

The measurement system based on machine vision of mechanical parts with multihole provide a new way to improved the efficiency of size measurement. To make the assembly line of mechanical parts run effectively, measurement system have to keep excellent connection with its both prior and next operation units. The experiment results show that the system have the advantages of high measurement accuracy and easy to realize automation, and therefore have broad market prospects.

\section{Acknowledgements}

This paper is partly supported by Guangdong province science and technology projects(2016A010106005, 2016B090918100), Zhongshan science and technology project (2017G1FC0009).

\section{References}

[1] Li Yunfeng, Han Cece, Li Shengyang. Measurement of dimension parameters of stamping parts based on machine vision [J]. Tool technology, 2015, 49 (1):95-98.

[2] Tang Bo, Kong Jianyi Overview of machine vision surface defect detection [J]. Journal of Image and Graphics, 2017, 22 (12): 1640-1663.
[3] Zhang Tian, Tang Chengtong, Liu Zhenhua. Measurement method of elbow space parameters based on multi-ocular vision [J]. Journal of instrumentation, 2013, 34 (2): 260-266.

[4] Wang Zhongfei, Lin Maosong, Peng Yong, etc. Microchip thickness detection based on machine vision [J]. Computer measurement and control, 2013, 21 (1): 36-38.

[5] Marco San Biagio, Carlos Beltrán-González, Salvatore Giunta, Alessio Del Bue, Vittorio Murino. Automatic inspection of aeronautic components [J]. Machine Vision and Applications. August 2017, Volume 28, Issue 5-6, pp 591-605.

[6] Luo Shiguang. Machine vision based inspection method for glass bottle mouth defects [J]. Packaging engineering, 2018, 39 (3):183-187

[7] Dan Zhongde, Zhang Fei. Research on on-line measurement technology of brinell hardness of machine vision casting [J]. Journal of mechanical engineering, 2017, 53 (1): 157-164.

[8] Li Jifeng, Zhao Fengxia, Jin Sahobo. Design of machine vision defect detection system for glass fiber cloth [J]. Machinery Design and Manufacture, 2018, 1 (1): 163-169.

[9] GUO Ruifeng, YUAN Chaofeng, YANG Liu, PENG Guangyu. Research on measurement of machine vision based on Open CV [J]. Computer Engineering and Applications, 2017, 53 (9):253-257.

[10] LI Yarrfeng, LI Xuyu, YANU liu. Rapid Glass Refractive Index Measurement Method Based on Machine Vision [J]. Acta Optica Sinica, 2017, 46 (11): 1-7. 